\title{
Exploiting wireless interference in heterogeneous networks
}

\author{
Wei, Zhongxiang, Masouros, Christos
}

Zhongxiang Wei, Christos Masouros, "Exploiting wireless interference in heterogeneous networks," Proc. SPIE 11711, Broadband Access Communication Technologies XV, 117110L (5 March 2021); doi: 10.1117/12.2584735

\section{SPIE. Event: SPIE OPTO, 2021, Online Only}




\title{
Exploiting Wireless Interference in Heterogeneous Networks
}

\author{
Zhongxiang Wei and Christos Masouros ${ }^{\mathrm{a}}$ \\ aDepartment of EEE, University College London
}

\begin{abstract}
In this work, the possibility of interference exploitation (IE) in heterogeneous networks is investigated. Based on the shared channel state information (CSI) and the transmitted data among the coordinated heterogeneous base stations (BS)s, we first formulate an IE-based coordinated multi-point (CoMP) scheme, which is able to utilize inter-cell interference as well as multiuser interference as constructive elements. Considering the effect of imperfect CSI at the BSs, we then minimize the transmission power consumption subject to users' statistical signal-to-interference-and-noise ratio (SINR) requirements. Simulation verifies that the proposed IE-based heterogeneous CoMP scheme significantly outperforms the benchmarks in terms of low power consumption.
\end{abstract}

Keywords: Heterogeneous Networks, Interference Exploitation, Precoding Design

\section{INTRODUCTION}

Inter-cell and intra-cell (multiuser) interference are two key impairments in the coming heterogeneous networks. ${ }^{1}$ A series of techniques has been demonstrated to suppress inter-cell and multiuser interference for enhancing downlink performance. Among them, coordinated multi-point (CoMP) has attracted much attention, which enables the heterogeneous base stations (BS)s to operate joint signal processing, based on the shared channel state information (CSI) and the data to be transmitted. ${ }^{2}$ Hence, CoMP heterogeneous networks in fact act as virtual multi-user multiple-input and multiple-output (MIMO), where all the channels (including the inter-cell ones) are able to carry signal. ${ }^{3}$ In the past decades, extensive researches have been conducted. $\mathrm{In},{ }^{4}$ a joint optimization design was demonstrated in heterogeneous CoMP networks, where each user is associated with one local macro BS and multiple distributed micro BSs. In, ${ }^{5}$ non-orthogonal multiple access was applied into CoMP networks, where the edge-cell users can be served by their adjacent BSs. In, ${ }^{6}$ beamforming was investigated under imperfect CSI, where the heterogeneous BSs can serve downlink user through the virtual multi-user multipleinput single-output (MISO) channel. From the view of information theory, heterogeneous CoMP transmission can be interpreted as a broadcast channel, where the multiuser interference should be suppressed as much as possible.

Conventional methods cancel multi-user interference by time division multiple access (TDMA), frequency division multiple access (FDMA), or spatial multiple access (SMA). As each user is allocated with part of resources, system performance of these orthogonality-based techniques is limited. In this case, the concept of interference exploitation (IE) has been proposed to utilize interference as a "green source" instead of suppressing it. ${ }^{1}$ IE was firstly introduced $b y^{7}$ in code division multiple access systems. Then ${ }^{8}$ further proposed that all the interference can be constructive by designing precoding in symbol level. ${ }^{9}$ Recently, the concept of IE was applied into beamforming optimization, ${ }^{10}$ physical layer security-aware systems, ${ }^{11}$ IoT, ${ }^{12}$ and multi-cell communications. ${ }^{9}$ However, it is noted that the existing IE-related techniques are designed for single-cell systems, which may not be directly applied to heterogeneous CoMP scenario. In particular, taking into consideration of imperfect CSI, how to guarantee multiple users' SINR requirements under infinite possibilities of CSI error imposes a new challenge. To address the open challenges, in this work, we investigate IE based CoMP heterogeneous schemes to exploit both inter-cell and multi-user interference as constructive elements. With CSI estimation errors at the BSs, we investigate the total power minimization problem in a probabilistic manner. Explicitly, the multiple users' SINR requirements are issued by statistical outage-probability constraints.

Further author information: Zhongxiang Wei: E-mail: zhongxiang.wei@ucl.ac.uk, and Christos Masouros: E-mail: c.masouros@ucl.ac.uk. This work was supported by the EPSRC Project EP/R007934/1.

Broadband Access Communication Technologies XV, edited by Benjamin B. Dingel, Katsutoshi Tsukamoto,

Spiros Mikroulis, Proc. of SPIE Vol. 11711, 117110L · C 2021 SPIE

CCC code: $0277-786 \mathrm{X} / 21 / \$ 21 \cdot$ doi: $10.1117 / 12.2584735$

Proc. of SPIE Vol. 11711 117110L-1 
Notations: Matrices and vectors are represented by boldface capital and lower case letters, respectively. $|\cdot|$ denotes the absolute value of a complex scalar. $\boldsymbol{A}^{H} \boldsymbol{A}^{T}$ and $\operatorname{Tr}(\boldsymbol{A})$ denote the Hermitian transpose, transpose and trace of matrix $\boldsymbol{A}$. $\boldsymbol{A} \succeq 0$ means $\boldsymbol{A}$ is a positive semi-definite matrix. Superscript $\Re$ and $\Im$ denote the real and imaginary parts, respectively.

\section{SYSTEM MODEL AND CONSTRUCTIVE INTERFERENCE}

In this section, system model is first introduced and then the concept of IE is discussed.

\subsection{System Model}

We consider a heterogeneous CoMP system at downlink, where the BSs exchange the CSI and the transmitted signal for coordination. Assume there are $N_{B S}$ BSs and $K$ users in each cell. Assume each BS is equipped with $M$ antennas, while each user is equipped with one antenna for simplicity. CSI is obtained by channel estimation in the training phase, based on channel reciprocity as in. ${ }^{13,14}$ By classic channel estimation methods, such as minimum mean square error (MMSE), the channel estimation error is modeled by the uncertainty-unbounded ${ }^{14}$ model. Define $\mathrm{U}_{i k}$ as the $k$-th user located in the $i$-th cell. Then its received signal is expressed as

$$
y_{i k}=\boldsymbol{h}_{i i k}^{T} \sum_{n=1}^{K} \boldsymbol{w}_{i n} s_{i n}+\sum_{j \neq i}^{N_{B S}} \sum_{m=1}^{K} \boldsymbol{h}_{j i k}^{T} \boldsymbol{w}_{j m} s_{j m}+n_{i k},
$$

where $h_{j i k} \in \mathbb{C}^{M \times 1}$ represents the channel from the $j$-th BS to the user $\mathrm{U}_{i k} . w_{i n} \in \mathbb{C}^{M \times 1}$ and $s_{\text {in }}$ denote the precoding and transmitted data at the $i$-th $\mathrm{BS}$ to the user $\mathrm{U}_{i n} . n_{i k} \in \mathbb{C}$ denotes the circular symmetric complex Gaussian noise at the user $\mathrm{U}_{i k}$, following $n_{i k} \sim \mathbb{C N}\left(0, \sigma_{n}^{2}\right), \forall i \in N_{B S}$ and $\forall k \in K$. Define $\mathbb{I}$ and $\mathbb{J}$ as the sets of users belonging to the $i$-th and $j$-th BSs, respectively. By conventional CoMP, the SINR at the user is given as

$$
\Gamma_{i k}^{\mathrm{CoMP}}=\frac{\sum_{j=1}^{N_{B S}}\left|\boldsymbol{h}_{j i k} \boldsymbol{w}_{i k}\right|^{2}}{\sum_{j=1}^{N_{B S}} \sum_{k^{\prime} \neq k, k^{\prime} \in \mathbb{J}}\left|\boldsymbol{h}_{j i k^{\prime}} \boldsymbol{w}_{i k^{\prime}}\right|^{2}+\sigma_{n}^{2}},
$$

where the first term in the denominator denotes the multiuser interference.

\subsection{The Concept of Interference Exploitation}

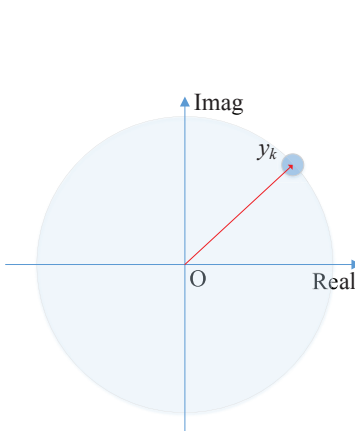

(a)

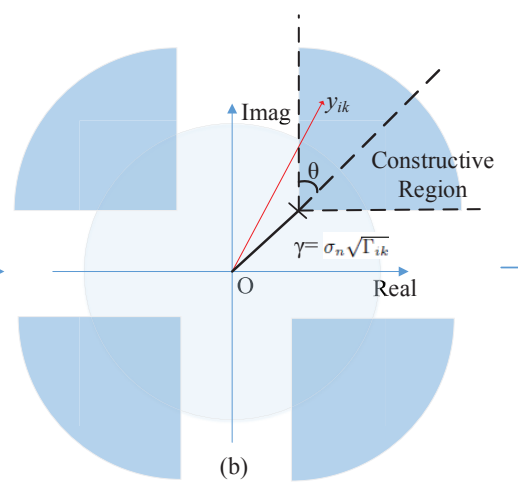

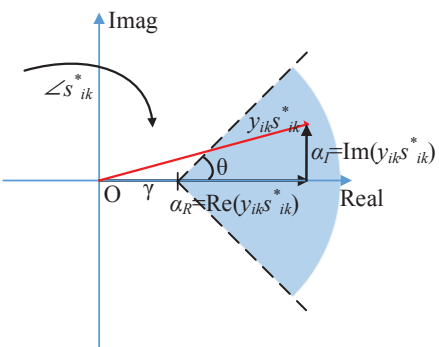

(c)

Figure 1. IE with QPSK. a) Conventional precoding locates symbols into a proximity region around the constellation point. b) By IE, the received signal $y_{i k}$ falls into a constructive region (dark blue area). The design pushes the resultant symbol away from the original decision threshold of the constellation, where $\gamma=\sigma_{n} \sqrt{\Gamma_{i k}}$. c) Rotated by $\angle s_{i k}^{*}$, the signal (noise excluded) is projected on real axis $\alpha_{R}=\Re\left\{y_{i k} s_{i k}^{*}\right\}$ and imaginary axis $\alpha_{I}=\Im\left\{y_{i k} s_{i k}^{*}\right\}$. According to the geometric interpretation, the received signal falls into constructive region (in Fig. 1 (b)) as long as the inequality $\left|\alpha_{I}\right| \leq\left(\alpha_{R}-\gamma\right) \tan \theta$ holds (in Fig. 1 (c)), where $\theta=\pi / Q$ and $Q$ denotes constellation size. 
By the concept of IE, interference can be exploited to push the received signals away from the detection thresholds of the signal constellation, and thus the increased distance to the detection threshold can effectively improve the receiver-side performance. Since the transmitted symbol can be written as $s_{i k}=d_{i k} e^{j\left(\phi_{i k}\right)}$, for the purpose of analysis we can express the symbol by another symbol such that $s_{i n}=s_{i k} e^{j\left(\phi_{i n}-\phi_{i k}\right)}$. Hence, the received signal in (1) is re-given as

$$
\left.y_{i k}=\boldsymbol{h}_{i i k}^{T} \sum_{n=1}^{K} \boldsymbol{w}_{i n} e^{\left(\phi_{i n}-\phi_{i k}\right)} s_{i k}+\sum_{j \neq i}^{N_{b s}} \sum_{m=1}^{K} \boldsymbol{h}_{j i k}^{T} \boldsymbol{w}_{j m} e^{\left(\phi_{j m}-\phi_{i k}\right.}\right) s_{i k}+n_{i k} .
$$

Define $\tilde{\boldsymbol{h}}_{j i k}=\boldsymbol{h}_{j i k} e^{j\left(\phi_{11}-\phi_{i k}\right)}$, and $\boldsymbol{w}_{j}=\sum_{m=1}^{K} \boldsymbol{w}_{j m} e^{j\left(\phi_{j m}-\phi_{11}\right)}, \forall j \in N_{B S}$. Note that the phase $\phi_{11}$ of the reference symbol $s_{11}$ can be arbitrary. Now, the received signal at the user $\mathrm{U}_{i k}$ is given as

$$
y_{i k}=\sum_{j=1}^{N_{B S}} \tilde{\boldsymbol{h}}_{j i k}^{T} \boldsymbol{w}_{j} s_{i k}+n_{i k}
$$

which essential indicates that the broadcast channel can be transformed into a virtual multicast channel with common messages to all users. Hence, by using the geometrical interpretation in Fig. 1, the requirement of IE is written as

$$
\left|\Im\left(\sum_{j=1}^{N_{B S}} \tilde{\boldsymbol{h}}_{j i k}^{T} \boldsymbol{w}_{j}\right)\right| \leq\left(\Re\left(\sum_{j=1}^{N_{B S}} \tilde{\boldsymbol{h}}_{j i k}^{T} \boldsymbol{w}_{j}\right)-\sigma_{n} \sqrt{\Gamma_{i k}}\right) \tan \theta, \forall \mathrm{U}_{i k},
$$

where the SINR requirement is also ensured.

\section{IE BASED HETEROGENEOUS COMP DESIGN UNDER IMPERFECT CSI}

Write the channel from the $j$-th BS to the user $\mathrm{U}_{i k}$ as $\tilde{\boldsymbol{h}}_{j i k}=\hat{\boldsymbol{h}}_{j i k}+\boldsymbol{e}_{i k}, \forall k \in K, j \in N_{B S} . \hat{\boldsymbol{h}}_{j i k} \in \mathbb{C}^{M \times 1}$ denotes the estimated channel with estimation error $\boldsymbol{e}_{i k} \in \mathbb{C}^{M \times 1}$. We adopt the uncertainty-unbounded model for the channel estimation error, where the element of channel estimation error vector follows Gaussian distribution such that $\left[\boldsymbol{e}_{i k}\right]_{m} \sim \mathbb{C N}\left\{0, \sigma_{i k}^{2}\right\}, \forall m \in M .^{14}$

\subsection{Problem Formulation}

Define precoding vector $\boldsymbol{w}_{j} \in \mathbb{C}^{M \times 1}, \forall j \in \mathbb{N}_{B S}$, as the precoding vector at the $j$-th BS and $\mathbb{N}_{B S}$ as the set of the coordinated BSs. The optimization problem can be given as

$$
\begin{aligned}
& P 1(\text { IE }- \text { Het - CoMP }): \underset{\boldsymbol{w}_{\boldsymbol{j}}, \forall j \in \mathbb{N}_{B S}}{\operatorname{argmin}} \sum_{j=1}^{N_{B S}}\left\|\boldsymbol{w}_{j}\right\|^{2}, \\
& \text { s.t }(C 1):\left\|\boldsymbol{w}_{j}\right\|^{2} \leq p_{\max }, \forall j \in \mathbb{N}_{B S}, \\
& \quad(C 2): \operatorname{Pr}\left\{\Gamma_{i k} \geq \bar{\Gamma}_{i k} \mid \boldsymbol{e}_{i k}\right\} \geq \eta_{i k}, \forall \mathrm{U}_{i k},
\end{aligned}
$$

where $\bar{\Gamma}_{i k}$ denotes the SINR requirement for the user $\mathrm{U}_{i k} . \eta_{i k}$ denotes the probabilistic threshold for the user $\mathrm{U}_{i k}$. Evidently, $(C 1)$ imposes individual transmission power constraint at each BS. $(C 2)$ guarantees that the SINR constraints at the user with probabilities $\eta_{i k}$ greater than the SINR requirement $\bar{\Gamma}_{i k}$. 


\subsection{Solution to the Optimization}

We first handle the statistical constraint $(C 2)$. Under the provision of IE, it equals to

$$
\begin{aligned}
& (C 2): \operatorname{Pr}\left\{\Gamma_{i k} \geq \bar{\Gamma}_{i k} \mid \boldsymbol{e}_{i k}\right\} \geq \eta_{i k} \stackrel{(5)}{\Rightarrow} \\
& \quad \operatorname{Pr}\left\{\left|\sum_{j=1}^{N_{B S}}\left(\tilde{\boldsymbol{h}}_{j i k}^{\Re}\right)^{T} \boldsymbol{w}_{j}^{\Im}+\left(\tilde{\boldsymbol{h}}_{j i k}^{\Im}\right)^{T} \boldsymbol{w}_{j}^{\Re}\right| \leq\left(\sum_{j=1}^{N_{B S}}\left(\left(\tilde{\boldsymbol{h}}_{j i k}^{\Re}\right)^{T} \boldsymbol{w}_{j}^{\Re}-\left(\tilde{\boldsymbol{h}}_{j i k}^{\Im}\right)^{T} \boldsymbol{w}_{j}^{\Im}\right)-\sigma_{n} \sqrt{\bar{\Gamma}_{i k}}\right) \tan \theta \mid \boldsymbol{e}_{i k}\right\} \geq \eta_{i k},
\end{aligned}
$$

Removing the absolute operator in (7) yields

$$
\left\{\begin{array}{l}
\operatorname{Pr}\left\{\sum_{j=1}^{N_{B S}}\left(\left(\tilde{\boldsymbol{h}}_{j i k}^{\Re}\right)^{T} \boldsymbol{w}_{j}^{\Im}+\left(\tilde{\boldsymbol{h}}_{j i k}^{\Im}\right)^{T} \boldsymbol{w}_{j}^{\Re}\right) \leq\left(\sum_{j=1}^{N_{B S}}\left(\left(\tilde{\boldsymbol{h}}_{j i k}^{\Re}\right)^{T} \boldsymbol{w}_{j}^{\Re}-\left(\tilde{\boldsymbol{h}}_{j i k}^{\Im}\right)^{T} \boldsymbol{w}_{j}^{\Im}\right)-\sigma_{n} \sqrt{\bar{\Gamma}_{i k}}\right) \tan \theta \mid \boldsymbol{e}_{i k}\right\} \geq \eta_{i k}, \\
\operatorname{Pr}\left\{-\sum_{j=1}^{N_{B S}}\left(\left(\tilde{\boldsymbol{h}}_{j i k}^{\Re}\right)^{T} \boldsymbol{w}_{j}^{\Im}+\left(\tilde{\boldsymbol{h}}_{j i k}^{\Im}\right)^{T} \boldsymbol{w}_{j}^{\Re}\right) \leq\left(\sum_{j=1}^{N_{B S}}\left(\left(\tilde{\boldsymbol{h}}_{j i k}^{\Re}\right)^{T} \boldsymbol{w}_{j}^{\Re}-\left(\tilde{\boldsymbol{h}}_{j i k}^{\Im}\right)^{T} \boldsymbol{w}_{j}^{\Im}\right)-\sigma_{n} \sqrt{\overline{\Gamma_{i k}}}\right) \tan \theta \mid \boldsymbol{e}_{i k}\right\} \geq \eta_{i k} .
\end{array}\right.
$$

The first inequality can be expanded into

$$
\operatorname{Pr}\left\{\sum_{j=1}^{N_{B S}}\left(\left[\hat{\boldsymbol{h}}_{j i k}^{\Im}+\boldsymbol{e}_{i k}^{\Im}-\hat{\boldsymbol{h}}_{j i k}^{\Re} \tan \theta-\boldsymbol{e}_{i k}^{\Re} \tan \theta ; \hat{\boldsymbol{h}}_{j i k}^{\Re}+\boldsymbol{e}_{i k}^{\Re}+\hat{\boldsymbol{h}}_{j i k}^{\Im} \tan \theta+\boldsymbol{e}_{i k}^{\Im} \tan \theta\right]^{T}\left[\boldsymbol{w}_{j}^{\Re} ; \boldsymbol{w}_{j}^{\Im}\right]\right) \leq-\sigma_{n} \sqrt{\bar{\Gamma}_{i k}} \tan \theta\right\} \geq \eta_{i k} .
$$

For simplicity, we rewrite (9) as $\operatorname{Pr}\left\{\sum_{j=1}^{N_{B S}}\left(\boldsymbol{a}_{j i k}^{T}\left[\boldsymbol{w}_{j}^{\Re} ; \boldsymbol{w}_{j}^{\Im}\right]\right) \leq-\sigma_{n} \sqrt{\bar{\Gamma}_{i k}} \tan \theta \mid \boldsymbol{e}_{i k}\right\} \geq \eta_{i k}$, where $\boldsymbol{a}_{j i k}=\left[\hat{\boldsymbol{h}}_{j i k}^{\Im}+\right.$ $\left.\boldsymbol{e}_{i k}^{\Im}-\hat{\boldsymbol{h}}_{j i k}^{\Re} \tan \theta-\boldsymbol{e}_{i k}^{\Re} \tan \theta ; \hat{\boldsymbol{h}}_{j i k}^{\Re}+\boldsymbol{e}_{i k}^{\Re}+\hat{\boldsymbol{h}}_{j i k}^{\Im} \tan \theta+\boldsymbol{e}_{i k}^{\Im} \tan \theta\right]$. It is easy to obtain that the $2 M$-dimensional Gaussian distributed vector $\boldsymbol{a}_{j i k}$ 's expectation is $\overline{\boldsymbol{a}}_{j i k}=\left[\hat{\boldsymbol{h}}_{j i k}^{\Im}-\hat{\boldsymbol{h}}_{j i k}^{\Re} \tan \theta ; \hat{\boldsymbol{h}}_{j i k}^{\Re}+\hat{\boldsymbol{h}}_{j i k}^{\Im} \tan \theta\right]$ with covariance matrix $\boldsymbol{\Theta}_{j i k}=$ $\operatorname{diag}(\underbrace{\left(1+\tan ^{2} \theta\right) \sigma_{i k}^{2}, \ldots,(1+\tan \theta)^{2} \sigma_{i k}^{2}}_{2 M})$. We now stack the vectors $\boldsymbol{w}_{j}$ and $\boldsymbol{a}_{j i k}, \forall j \in \mathbb{N}_{B S}$, into two long vectors $\boldsymbol{w}$ and $\boldsymbol{a}_{i k}$, such that $\boldsymbol{w}=\operatorname{vec}\left(\boldsymbol{w}_{1}^{\Re} ; \boldsymbol{w}_{1}^{\Im} ; \boldsymbol{w}_{2}^{\Re} ; \boldsymbol{w}_{2}^{\Im} ; \ldots ; \boldsymbol{w}_{N_{B S}}^{\Re} ; \boldsymbol{w}_{N_{B S}}^{\Im}\right)$ and $\boldsymbol{a}_{i k}=\operatorname{vec}\left(\boldsymbol{a}_{1 i k} ; \boldsymbol{a}_{1 i k} ; \ldots ; \boldsymbol{a}_{N_{B S} i k}\right)$. It is observed that the long vector $\boldsymbol{a}_{i k}$ 's expectation is calculated as $\overline{\boldsymbol{a}}_{i k}=\operatorname{vec}\left(\overline{\boldsymbol{a}}_{1 i k} ; \overline{\boldsymbol{a}}_{1 i k} ; \ldots ; \overline{\boldsymbol{a}}_{N_{B S} i k}\right)$ with covariance matrix $\boldsymbol{\Theta}_{i k}=\operatorname{diag}(\underbrace{\left(1+\tan ^{2} \theta\right) \sigma_{i k}^{2}, \ldots,\left(1+\tan ^{2} \theta\right) \sigma_{i k}^{2}}_{2 M \times N_{B S}})$. Now (9) can be derived as

$$
\begin{aligned}
& \operatorname{Pr}\left\{\boldsymbol{a}_{i k}^{T}\left[\boldsymbol{w}_{1}^{\Re} ; \boldsymbol{w}_{1}^{\Im} ; \ldots ; \boldsymbol{w}_{N_{B S}}^{\Re} ; \boldsymbol{w}_{N_{B S}}^{\Im}\right] \leq-\sigma_{n} \sqrt{\bar{\Gamma}_{i k}} \tan \theta \mid \boldsymbol{e}_{i k}\right\} \geq \eta_{i k} \\
& \Rightarrow \Phi\left(\frac{-\sigma_{n} \sqrt{\bar{\Gamma}_{i k}} \tan \theta-\overline{\boldsymbol{a}}_{i k}^{T}\left[\boldsymbol{w}_{1}^{\Re} ; \boldsymbol{w}_{1}^{\Im} ; \ldots ; \boldsymbol{w}_{N_{B S}}^{\Re} ; \boldsymbol{w}_{N_{B S}}^{\Im}\right]}{\|\left.\Theta_{i k}^{\frac{1}{2}}\left[\boldsymbol{w}_{1}^{\Re} ; \boldsymbol{w}_{1}^{\Im} ; \ldots ; \boldsymbol{w}_{N_{B S}}^{\Re} ; \boldsymbol{w}_{N_{B S}}^{\Im}\right]\right|_{2}}\right) \geq \eta_{i k},
\end{aligned}
$$

where $\Phi(x)=\frac{1}{\sqrt{2 \pi}} \int_{-\infty}^{x} e^{\frac{-t^{2}}{2}} \mathrm{~d} t$ denotes the cumulative probability function (cdf) of a standard Gaussian distributed variable. Defining $\Phi^{-1}(\cdot)$ as the inverse function of $\Phi(\cdot),(10)$ can be finally derived into a quadratic constraint such that

$$
\overline{\boldsymbol{a}}_{i k}^{T}\left[\boldsymbol{w}_{1}^{\Re} ; \boldsymbol{w}_{1}^{\Im} ; \ldots ; \boldsymbol{w}_{N_{B S}}^{\Re} ; \boldsymbol{w}_{N_{B S}}^{\Im}\right]+\Phi^{-1}\left(\eta_{i k}\right)\left\|\boldsymbol{\Theta}_{i k, 1}^{\frac{1}{2}}\left[\boldsymbol{w}_{1}^{\Re} ; \boldsymbol{w}_{1}^{\Im} ; \ldots ; \boldsymbol{w}_{N_{B S}}^{\Re} ; \boldsymbol{w}_{N_{B S}}^{\Im}\right]\right\|_{2} \leq-\sigma_{n} \sqrt{\bar{\Gamma}_{i k}} \tan \theta .
$$

Similarly, the second inequality in (8) can be given as

$$
\overline{\boldsymbol{b}}_{i k}^{T}\left[\boldsymbol{w}_{1}^{\Re} ; \boldsymbol{w}_{1}^{\Im} ; \ldots ; \boldsymbol{w}_{N_{B S}}^{\Re} ; \boldsymbol{w}_{N_{B S}}^{\Im}\right]+\Phi^{-1}\left(\eta_{i k}\right)\left\|\boldsymbol{\Theta}_{i k}^{\frac{1}{2}}\left[\boldsymbol{w}_{1}^{\Re} ; \boldsymbol{w}_{1}^{\Im} ; \ldots ; \boldsymbol{w}_{N_{B S}}^{\Re} ; \boldsymbol{w}_{N_{B S}}^{\Im}\right]\right\|_{2} \leq-\sigma_{n} \sqrt{\bar{\Gamma}_{i k}} \tan \theta,
$$

where $\overline{\boldsymbol{b}}_{i k}=\operatorname{vec}\left(\overline{\boldsymbol{b}}_{1 i k} ; \overline{\boldsymbol{b}}_{2 i k} ; \ldots ; \overline{\boldsymbol{b}}_{N_{B S} i k}\right)$ and $\overline{\boldsymbol{b}}_{j i k}=\left[-\hat{\boldsymbol{h}}_{j i k}^{\Im}-\hat{\boldsymbol{h}}_{j i k}^{\Re} \tan \theta ;-\hat{\boldsymbol{h}}_{j i k}^{\Re}+\hat{\boldsymbol{h}}_{j i k}^{\Im} \tan \theta\right]$ with covariance matrix calculated as $\boldsymbol{\Theta}_{i k}=\operatorname{diag}(\underbrace{(1+\tan \theta)^{2} \sigma_{i k}^{2}, \ldots,(1+\tan \theta)^{2} \sigma_{i k}^{2}}_{2 M \times N_{B S}})$. Now, constraint $(C 2)$ has been transformed into the 
two inequalities in (11) and (12). According to Schur Complements that $\|\boldsymbol{A} \boldsymbol{x}+\boldsymbol{b}\|_{2} \leq \boldsymbol{e}^{T} \boldsymbol{x}+d$ being equivalent to $\left[\begin{array}{cc}\left(\boldsymbol{e}^{T} \boldsymbol{x}+d\right) \boldsymbol{I} & \boldsymbol{A} \boldsymbol{x}+\boldsymbol{b} \\ (\boldsymbol{A} \boldsymbol{x}+\boldsymbol{b})^{T} & \boldsymbol{e}^{T} \boldsymbol{x}+d\end{array}\right] \succeq \mathbf{0}$, the above two inequalities in (11) and (12) can be further transformed into two linear matrix inequalities (LMI) as

$$
\left\{\begin{array}{c}
{\left[\begin{array}{cc}
\frac{\left(-\overline{\boldsymbol{a}}_{i k}^{T}\left[\boldsymbol{w}_{1}^{\Re} ; \boldsymbol{w}_{1}^{\Im} ; \ldots ; \boldsymbol{w}_{N_{B S}}^{\Re} ; \boldsymbol{w}_{N_{B S}}^{\Im}\right]-\sigma_{n} \sqrt{\bar{\Gamma}_{i k}} \tan \theta\right) \boldsymbol{I}}{\Phi-1\left(\eta_{i k}\right)} & \Theta_{i k}^{\frac{1}{2}}\left[\boldsymbol{w}_{1}^{\Re} ; \boldsymbol{w}_{1}^{\Im} ; \ldots ; \boldsymbol{w}_{N_{B S}}^{\Re} ; \boldsymbol{w}_{N_{B S}}^{\Im}\right] \\
\left(\Theta_{i k}^{\frac{1}{2}}\left[\boldsymbol{w}_{1}^{\Re} ; \boldsymbol{w}_{1}^{\Im} ; \ldots ; \boldsymbol{w}_{N_{B S}}^{\Re} ; \boldsymbol{w}_{N_{B S}}^{\Im}\right]\right)^{T} & \frac{-\overline{\boldsymbol{a}}_{i k}^{T}\left[\boldsymbol{w}_{1}^{\Re} ; \boldsymbol{w}_{1}^{\Im} ; \ldots ; \boldsymbol{w}_{N_{B S}}^{\Re} \boldsymbol{w}_{N_{B S}}^{\Im}\right]-\sigma_{n} \sqrt{\bar{\Gamma}_{i k}} \tan \theta}{\Phi^{-1}\left(\eta_{i k}\right)}
\end{array}\right] \succeq \mathbf{0},} \\
{\left[\begin{array}{cc}
\frac{\left(-\overline{\boldsymbol{b}}_{i k}^{T}\left[\boldsymbol{w}_{1}^{\Re} ; \boldsymbol{w}_{1}^{\Im} ; \ldots ; \boldsymbol{w}_{N_{B S}}^{\Re} ; \boldsymbol{w}_{N_{B S}}^{\Im}\right]-\sigma_{n} \sqrt{\bar{\Gamma}_{i k}} \tan \theta\right) \boldsymbol{I}}{\Phi^{-1}\left(\eta_{i k}\right)} & \Theta_{i k}^{\frac{1}{2}}\left[\boldsymbol{w}_{1}^{\Re} ; \boldsymbol{w}_{1}^{\Im} ; \ldots ; \boldsymbol{w}_{N_{B S}}^{\Re} ; \boldsymbol{w}_{N_{B S}}^{\Im}\right] \\
\left(\Theta_{i k}^{\frac{1}{2}}\left[\boldsymbol{w}_{1}^{\Re} ; \boldsymbol{w}_{1}^{\Im} ; \ldots ; \boldsymbol{w}_{N_{B S}}^{\Re} ; \boldsymbol{w}_{N_{B S}}^{\Im}\right]\right)^{T} & \frac{-\overline{\boldsymbol{b}}_{i k}^{T}\left[\boldsymbol{w}_{1}^{\Re} ; \boldsymbol{w}_{1}^{\Im} ; \ldots ; \boldsymbol{w}_{N_{B S}}^{\Re} ; \boldsymbol{w}_{N_{B S}}^{\Im}\right]-\sigma_{n} \sqrt{\bar{\Gamma}_{i k}} \tan \theta}{\Phi^{-1}\left(\eta_{i k}\right)}
\end{array}\right] \succeq \mathbf{0}}
\end{array}\right.
$$

Defining $\boldsymbol{W}_{j}=\boldsymbol{w}_{j} \boldsymbol{w}_{j}^{H}, \forall j \in \mathbb{N}_{B S}$, P1 can be finally written as

$$
\begin{aligned}
& P 2(\mathrm{IE}-\text { Het }- \text { CoMP }): \underset{\boldsymbol{W}_{j}, j \in \mathbb{N}_{B S}}{\operatorname{argmin}} \sum_{j=1}^{N_{B S}} \operatorname{Tr}\left(\boldsymbol{W}_{j}\right), \\
& \text { s.t }(C 1): \operatorname{Tr}\left(\boldsymbol{W}_{j}\right) \leq p_{\max }, \forall j \in \mathbb{N}_{B S},(C 2):(13), \forall \mathrm{U}_{i k},(C 3):\left[\begin{array}{cc}
\boldsymbol{W}_{j} & \boldsymbol{w}_{j} \\
\boldsymbol{w}_{j}^{T} & 1
\end{array}\right] \succeq 0, \forall j \in \mathbb{N}_{B S},
\end{aligned}
$$

which is a convex semi-definite programming (SDP) and can be readily solved by CVX solver.

\section{SIMULATION RESULTS}

We employ Monte Carlo simulations to verify the proposed design. The central frequency is set to $2 \mathrm{GHz}$ with $1 \mathrm{MHz}$ bandwidth. The AWGN power spectral density is $-174 \mathrm{dBm} / \mathrm{Hz}$. A 3-cell scenario is considered, and in each cell a BS is located in the center. The number of antennas at each BS is set to $M=4$. It is assumed there are $K=3$ users in each cell and are distributed randomly. QPSK is adopted as constellation scheme. The maximum transmission power is set to $p_{\max }=40 \mathrm{dBm}$. Without loss of generality, the outage probability factor (for the probabilistic manner optimization) and SINR requirement are set to $\eta_{i k}=80 \%$ and $\bar{\Gamma}_{i k}=20 \mathrm{~dB}$, respectively. The standard deviation of the CSI uncertainty is set to $\sigma_{i k}=10^{-2}$ for all the users. PL model in ${ }^{14}$ and Rayleigh fading are adopted for modelling channels. The following schemes are selected as benchmarks. a) CoMP with perfect $\mathrm{CSI}^{2}$ (Conv-Het-CoMP). b) Coordinated beamforming $(\mathrm{CBF}),{ }^{14}$ where the inter-cell interference is suppressed by the coordinated BSs.

Fig. 2 shows the impact of users' SINR requirements $\bar{\Gamma}_{i k}$ on the total power consumption. It can be seen that the proposed design consume the lowest power. It is because it utilizes both inter-cell and multi-user interference as constructive elements, enabling a significant power saving to achieve a target SINR. As comparison, although the Conv-Het-CoMP scheme works as a network-level MISO to obtain a broadcast channel, the multi-user interference still needs to be canceled. As a result, even with perfect CSI, it is still inferior to the proposed design. Also, since by the CBF algorithm, each BS only serves its local users and inter-cell channel is unable to transmit useful signal, it consumes the highest power among the algorithms.

\section{CONCLUSION}

In this paper, we have proposed a power-efficient IE-based robust precoder for heterogeneous CoMP systems, under the effect of CSI estimation error. By exploiting both inter-cell and multi-user interference as constructive elements, the proposed scheme presents the lowest power consumption, and even outperforms the conventional CoMP with perfect CSI acquisition. 


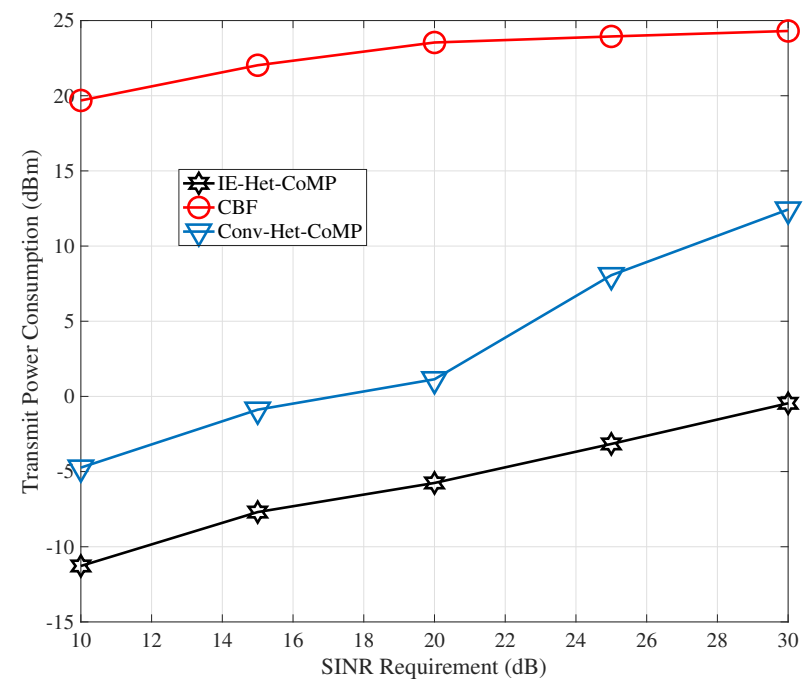

Figure 2. The impact of SINR requirement on power consumption performance.

\section{REFERENCES}

[1] Masouros, C., Rntnarajah, T., and Qinetiq, A. K. S., "Known interference in the cellular downlink: a performance limiting factor or a source of green signal power?," IEEE Commun. Mag. 51, 162-171 (2013).

[2] Lee, D., Seo, H., Clerckx, B., Hardouin, E., and Sayana, K., "Coordinated multipoint transmission and reception in lte-advanced: deployment scenarios and operational challenges," IEEE Commun. Mag. 50, 162-171 (2013).

[3] Gesbert, D., Hanly, S., Huang, H., Shitz, S. S., Simeone, O., and Yu, W., "Multi-cell mimo cooperative networks: a new look at interference," IEEE Journal on Sel. Area in Commun. 28, 1380-1408 (2010).

[4] Wang, F. and Wu, Q., "Joint optimization of user association, subchannel allocation, and power allocation in multi-cell multi-association ofdma heterogeneous networks," IEEE Trans. Commun. 65, 2672-2684 (2017).

[5] Ali, M., Hossain, E., Al-Dweik, A., and Kim, D. I., "Downlink power allocation for comp-noma in multi-cell networks," IEEE Trans. Commun. 66, 3982-3998 (2018).

[6] Ararat, S. and Nakhai, M. R., "Robust distributed beamforming with interference coordination in downlink cellular networks," IEEE Trans. Commun. 62, 2411-2421 (2014).

[7] Masouros, C. and Alsusa, E., "A novel transmitter-based selective precoding technique for ds/cdma systems," IEEE Sig. Process. Lett. 14, 637-640 (2007).

[8] Masouros, C., "Correlation rotation linear precoding for mimo broadcast communications," IEEE Trans. Signal Process. 59, 252-262 (2011).

[9] Wei, Z., Masouros, C., Wong, K. K., and Kang, X., "Multi-cell interference exploitation: enhancing the power efficiency in cell coordination," IEEE Trans. Wireless Commun. 19, 547-562 (2020).

[10] Masouros, C. and Zheng, G., "Vector perturbation based on symbol scaling for limited feedback miso downlinks," IEEE Trans. Sig. Proc. 63, 3668-3680 (2015).

[11] Wei, Z. and Masouros, C., "Device-centric distributed antenna transmission: secure precoding and antenna selection with interference exploitation," IEEE Internet Things J. 7, 2293-2308 (2020).

[12] Wei, Z., Masouros, C., Liu, F., Chatzinotas, S., and Ottersten, B., "Energy- and cost-efficient physical layer security in the era of iot: the role of interference," IEEE Commun. Mag. 1, 81-88 (2020).

[13] Wei, Z., Sun, S., Zhu, X., Huang, Y., and Wang, J., "Energy-efficient hybrid duplexing strategy for bidirectional distributed antenna systems," IEEE Trans. Veh. Technol. 67, 5096-5110 (2018).

[14] Nasseri, S., Nakhai, M. R., and Le, T. A., "Chance constrained robust downlink beamforming in multicell networks," IEEE Trans. Mobile. Comput. 15, 2682-2691 (2016). 